\title{
La conception de la « distance » de Jacques Henriot : un espace virtuel de jeu
}

\section{Maude Bonenfant}

\section{OpenEdition}

\section{Journals}

Édition électronique

URL : http://journals.openedition.org/sdj/235

DOI : $10.4000 /$ sdj.235

ISSN : 2269-2657

Éditeur

Laboratoire EXPERICE - Centre de Recherche Interuniversitaire Expérience Ressources Culturelles Education

\section{Référence électronique}

Maude Bonenfant, «La conception de la « distance » de Jacques Henriot : un espace virtuel de jeu », Sciences du jeu [En ligne], 1 | 2013, mis en ligne le 01 octobre 2013, consulté le 28 mars 2021. URL : http://journals.openedition.org/sdj/235; DOl : https://doi.org/10.4000/sdj.235

Ce document a été généré automatiquement le 28 mars 2021.

\section{(c) (i) (9)}

La revue Sciences du jeu est mise à disposition selon les termes de la Licence Creative Commons Attribution - Pas d'Utilisation Commerciale - Pas de Modification 4.0 International. 


\title{
La conception de la « distance » de Jacques Henriot : un espace virtuel de jeu
}

\author{
Maude Bonenfant
}

1 Jacques Henriot est l'un des auteurs sur le jeu les plus inspirants. À travers ses écrits, il propose une manière originale et cohérente de considérer l'activité ludique en divisant son étude en trois degrés d'analyse : 1) ce à quoi joue celui qui joue (la structure régulée $\mathrm{du}$ jeu) ; 2) ce que fait celui qui joue (l'acte de jouer) ; 3) ce qui fait que le joueur joue (ce pourquoi le jeu est rendu possible et réel grâce au joueur). De ces trois degrés (jeu, jouer et jouant), Henriot tire des questionnements : «pourquoi, dans tout jeu, il y a "jeu", [quels sont les] critères objectifs du jouer pris comme forme de conduite [et] à quelles conditions cet être peut en venir à jouer» (Henriot, 1969, p. 17) ? Dans cet article, nous voudrions proposer comme hypothèse que la réponse à ces questions se trouve dans la conception même de la distance, au cœur de la réflexion de Jacques Henriot sur le jeu, mais aussi au cœur de toute réflexion sur l'ontologie du jeu. ${ }^{1}$

\section{La conception de la distance chez Henriot}

Depuis Henriot et plusieurs autres auteurs sur le jeu (cf. Fink, 1965 ; Suits, 1990 ; Salen \& Zimmerman 2004), l'essence du jeu se comprend entre la structure (comme game) et l'entreprise abstraite qui donne vie au jeu (le play) en tant qu'attitude. Or, ce qu'il faut bien comprendre, c'est que malgré le caractère diachronique irréversible (processus orienté) du jeu (Henriot, 1969, pp. 29-31), s'immiscent de la contingence et des points d'indétermination aux carrefours du jeu. Tout jeu comprend une marge d'incertitude et d'indétermination, non pas nécessairement au niveau de l'objet-jeu, mais pour le sujetjoueur qui ignore l'ensemble des causes.

Cette marge, en théorie du moins, peut toujours être calculée comme l'effet mesurable d'un ensemble de causes : l'indétermination n'est pas le résultat d'une contingence réelle au niveau de l'objet, mais seulement la conséquence d'un état d'ignorance dans lequel se trouve le sujet à l'égard de l'objet (Henriot, 1969, p. 73). 
Cette contingence n'est pas inscrite dans le jeu, mais dans l'interprétation du jeu - dans les sens produits pour et par le joueur. Même si l'on pouvait supposer que tout est déterminé d'avance, le joueur ne peut pas connaître l'entièreté des causes, mais cette incertitude résulte des limites de l'intelligence humaine (Henriot, 1989, p. 240). Pour cette raison, Henriot affirme que « le hasard n'est que l'effet de l'ignorance » (1989, p. 241). Ainsi, dans certains jeux, comme le morpion (Tic-Tac-Toe), ${ }^{2}$ existe une part de contingence pour les néophytes et aucune contingence pour les habitués qui connaissent la logique du jeu parce que les règles ne se réduisent qu'à un seul sens : toutes les parties se termineront en partie nulle.

4 L'incertitude est donc plus dans l'état du joueur lui-même que dans l'ensemble des règles $\mathrm{du}$ jeu. Alors que l'on s'efforce habituellement de réduire au maximum l'imprévisibilité de la vie, le jeu en fait son moteur. «Jouer, c'est ne pas savoir où l'on va, même si l'on a soigneusement préparé son itinéraire et calculé ses effets » (Henriot, 1969, p. 76). C'est par le risque que le joueur sait ce dont il est capable et qu'il y a jeu. Il n'existe qu'à partir du moment où un joueur s'aventure dans l'imprévisible et y risque quelque chose (Henriot, 1989, p. 252).

5 Cependant, ces possibilités de faire tel ou tel choix sont en nombre limité, surtout dans les jeux très codifiés où ces choix sont définis (ludus). Le joueur ne peut y improviser que de manière réduite alors que les jeux moins codifiés (paidia) ${ }^{3}$ lui laissent une plus grande marge de manœuvre et des choix moins déterminés. Or, ces choix sont toujours limités, car des choix illimités seraient la négation même du jeu : « si, à chaque instant, chaque joueur avait le droit de faire n'importe quoi, le jeu perdrait de sa consistance » (Henriot, 1969, p. 32).

6 Ces balises ne sont pas le jeu. «Le jeu se joue du non-jeu : c'est en cela qu'il est jeu » (Henriot, 1969, p. 72). Jouer est une opération dialectique qui met à distance le joueur par rapport au non-jeu. La forme du jeu est cette distance, cet intervalle symbolique qui permet au jeu d'exister pour un joueur.

Le jouer, dans son essence (c'est-à-dire : selon l'idée que l'on s'en fait), tient à la marge de jeu, à la distance qui se crée et se maintient entre le joueur et son jeu, entre ce qu'il est et ce qu'il fait, entre le sujet et le verbe de l'énonciation: «je joue» (Henriot, 1989, p. 149).

7 Cette distance intérieure qui est mise en place par le joueur est ce qui différencie ce que le joueur fait par jeu ou non.

Seule la distance établie par le joueur entre ce qu'il fait et le fait de le faire par jeu, ce survol et ce contrôle de soi permet de caractériser de manière strictement subjective la conduite que l'on qualifie de ludique (Henriot, 1989, p. 256).

On se rend alors compte que le sens importe plus que la structure puisque, ultimement, le sens engendre la structure. Le jeu ne prend le sens de jeu que si quelqu'un se définit comme joueur éventuel. «Le jeu tient d'abord au jeu qu'il y a entre le joueur et son jeu » (Henriot, 1969, p. 66). Le sens du jeu est produit par celui qui joue (acte), grâce à son attitude ludique, ${ }^{4}$ mais ce sens est rendu possible par la distance du joueur face à son jeu (intention).

Qu'il s'agisse de jeu ou de jouet, ces réalités n'ont de sens et de fonction que parce qu'elles sont l'objet d'un jouer (play) qui tient lui-même au jeu que le joueur, par son attitude, introduit et maintient entre son jeu et lui (Henriot, 1969, p. 73).

9 Le jeu est à la fois conduite objectivement observable (l'ensemble de ses actes) et intention subjective de donner tel sens à une conduite. « Une situation d'incertitude n'a 
de valeur ludique qu'à partir du moment où quelqu'un décide de s'en jouer » (Henriot, 1989, p. 253). Cette intention de donner le sens de jeu à son acte interdit à l'observateur extérieur de voir du « jeu » chez un enfant, un animal ou même un autre être humain.

Le phénomène « jeu » appartient à l'ordre du signifiant. Ce qu'il y a de jeu dans un acte ludique relève uniquement du sens. Il a sens de jeu : à cela se ramène le fait qu'il soit jeu. On peut faire quelque chose sans jouer; on peut faire la même chose par jeu. La différence tient seulement au sens que l'on donne à son acte (Henriot, 1969, p. 48).

Henriot donne l'exemple des enfants qui s'amusent avec des cailloux: ces cailloux prennent alors un autre sens, celui du jeu, aussi longtemps que les enfants s'amusent puis ils redeviennent simples cailloux à partir du moment où les joueurs cessent leur jeu.

\section{La conception de la « distance » chez Henriot : une question de conscience}

11 Pour Henriot, le jeu est acte d'imagination de la part de l'interprète qui devient joueur. Le joueur s'imagine qu'il joue, en tant qu'idée : «le jeu, c'est de l'imaginaire en acte » (Henriot, 1989, p. 156). Henriot croit que la conscience du joueur dans l'exercice du jeu discriminerait ce qui est du jeu de ce qui ne l'est pas: « un jeu dont on n'est pas conscient n'est pas un jeu » (Henriot, 1969, p. 9). Il faut une prise de conscience que ceci est un jeu pour qu'il y ait jeu, mais, en même temps, dès que l'on prend conscience que ceci est un jeu, il n'y a plus jeu : en étant conscient du jeu, l'illusion est détruite et le jeu par le fait même. " Nul ne jouerait, bien sûr, s'il ne savait qu'il joue ; pourtant il paraît psychologiquement impossible de jouer et de penser qu'on joue, de jouer et de dire qu'on joue » (Henriot, 1989, p. 152). Henriot pose l'hypothèse que cette dialectique est peut-être l'essence du jeu.

Si matériel et structure (la chose avec laquelle on joue et la chose à quoi l'on joue) ne prennent signification et valeur ludiques qu'à partir du moment où quelqu'un décide d'y jouer ou de s'en servir pour jouer, il en résulte, semble-t-il, que la seule « chose » qui soit à définir quand on parle de jeu est la forme de pensée, l'attitude mentale, la conscience singulière de celui qui découvre dans ce matériel et dans cette structure des occasions ou des moyens de jouer (Henriot, 1989, p. 123).

La distance du jeu permettant la contingence est aussi celle du joueur lui-même par rapport à ce qu'il fait. "Tout se passe comme s'il se dédoublait, se voyait en train de faire ce qu'il fait, avec l'assurance qu'il ne s'agit que d'un jeu» (Henriot, 1969, p. 76). C'est une distanciation doublée d'une déréalisation : le joueur qui sait qu'il joue perd le contact avec le réel. Il y a illusion du jeu, c'est-à-dire une entrée dans le jeu.

Malgré le fait qu'il concède une réalité au jeu dans la conscience du joueur et la matérialité de l'action, Henriot parle du jeu comme le "on dirait», le "supposons » consciemment posé par le joueur. Le joueur doit être conscient de jouer et renouveler à chaque instant sa décision de jouer pour qu'il y ait jeu : « jouer c'est savoir qu'on joue » (Henriot, 1989, p. 147). La conscience de jouer est le passage dans ce que le joueur est conscient de faire par jeu, dans ce que l'auteur appelle le surréalisme. Henriot croit qu'il n'existe pas de jeu dont nous ne soyons pas conscients et qu'il y a toujours intention subjective de jouer. 

nous aimerions proposer une lecture différente de ce qu'est cette « distance » du jeu. Cette nouvelle interprétation ne vient pas en opposition avec l'approche de la conscience de Henriot, mais offre plutôt un nouvel éclairage sur cette conception de la « distance » sans référer à la conscience - qui peut être un concept mouvant dont l'emprise est difficile pour parler de l'expérience ludique.

\section{La conception de la " distance » chez Henriot : pour se sortir de la conscience}

Pour parler du concept de jeu en abordant la conception de la " distance ", il n'est pas nécessaire de l'inscrire dans la conscience du joueur. En fait, nous ne croyons pas qu'il soit essentiel de marquer l'unicité du sujet dans un être autoréflexif pour parler du jeu, même s'il est question du jeu de l'être en devenir. Preuve en est faite qu'Henriot luimême affirme qu'il y a jeu dans un engrenage ou un mécanisme. Il insiste même pour dire que c'est le sens premier du concept. Que l'individu interprète son action comme $\mathrm{du}$ jeu ou non, la fonction de jeu permet à l'interprète de définir le sens et de faire émerger de nouveaux sens au jeu. Ainsi compris, celui qui est un sportif professionnel ne joue pas, au sens de la notion de jeu, mais il y a quand même du jeu (mécanique) dans son interprétation des règles du jeu, dans sa manière de développer un style, dans les stratégies et tactiques utilisées, etc. Au contraire de ce que dit Henriot (1989, p. 260), jouer, c'est plus que "savoir que l'on joue " : il peut y avoir jeu sans que le joueur pose le cadre interprétatif « ceci est du jeu ». Si tout n'est pas du jeu, il peut y avoir une part de jeu dans toute activité, même si elles ne prennent pas le sens relatif et culturel de « jeu ».

En fait, avec sa conception de la «distance », Henriot définit deux choses à la fois, la notion et la fonction de jeu. Lorsqu'il définit le jeu à partir de la conscience du joueur, il définit la notion de jeu et elle est alors relative à un contexte (spatiotemporel, culturel, etc.). Or, lorsqu'il définit le jeu sur un plan mécanique, il définit alors la fonction de jeu. L'unité de sens, le dénominateur commun, ce noyau sémantique dont il parle, est la fonction de jeu, singulière et universelle, soit les conditions de possibilité du jeu.

17 Si le «jeu » n'est pas partout le même, Henriot affirme que les chercheurs emploient toujours le même mot et font référence à une seule et même idée. Il croit à une idée du jeu qui dépasse «les limites du relativisme historique et sociologique» (Henriot, 1989, p. 15) et à une sorte de signification transcendante prise dans son contexte. Ce " jeu » n'est pas dans le premier degré de l'acte, bien souvent différent, mais dans une signification seconde, une unicité de sens (à ranger avec les " universaux »).

Co idée universelle du jeu, que nous croyons être la fonction de jeu (Bonenfant, 2010), est représentée dans le mouvement des choses (des engrenages, des portes, des bateaux qui jouent sur leur ancre, etc.). Pour Henriot, le mouvement des choses est une image que l'humain utilise pour comprendre son propre jeu, mais nous croyons que ce mouvement des choses et entre les choses réfère à la fonction de jeu, c'est-à-dire que ce mouvement est une des conditions de possibilité du jeu. Le jeu mécanique n'est pas qu'une métaphore: il est ce par quoi tout jeu est possible. «Distinguer, dans [le domaine du jeu], l'emploi propre de l'emploi métaphorique, n'a finalement plus de sens » (Gadamer, 1960, p. 123). Il s'agira maintenant de définir ce jeu mécanique ou 
plutôt cette distance de Henriot qui est une des conditions de possibilité de l'activité ludique.

\section{La part virtuelle du jeu}

19 Henriot affirme que le jeu est «l'exercice du possible » $(1989$, p. 236). Nous ajoutons que « le jeu est exercice du possible et du virtuel », le virtuel étant entendu comme ce qui n'a pas lieu, qui est infini et indéfini (Bonenfant, 2011). Le jeu n'est pas seulement ce qui est défini, mais surtout ce qui est indéfini avant même l'expérience ludique. Dans tout jeu existe une part virtuelle qui ajoute à l'incertitude produite par la structure du jeu. Aucun jeu n'est entièrement défini, puisque ce serait la négation même du jeu. Le joueur peut s'approprier cette part virtuelle et créer des sens infinis et nouveaux. Cette part indéterminée et illimitée du jeu est, selon nous, la distance définie par Henriot.

Comme pour les possibles du jeu, la part de virtuel est faite d'incertitude. Or, celle-ci est dans le sens à donner aux actions et aux réponses produites par le jeu - au contraire de l'incertitude des possibles qui est une incertitude des choix et résultats. Par exemple, au poker, l'incertitude des possibles concerne les combinaisons de cartes: on peut, théoriquement, prédire l'ensemble des combinaisons possibles en comptant les cartes passées, par exemple. L'incertitude concerne donc les choix des joueurs (les cartes qu'ils conserveront et celles qu'ils échangeront) et les résultats parmi toutes ces combinaisons probables. L'incertitude issue de la part virtuelle du poker est, quant à elle, inscrite dans le sens global à donner au jeu, dans toutes ces tactiques des joueurs pour donner de faux indices, pour jouer de manière inhabituelle, pour lire les indices que les autres joueurs laissent transparaître, etc. Si l'incertitude des possibles est encadrée (par les probabilités, par exemple), l'incertitude de la part virtuelle est une ouverture vers l'infini par la création de nouveaux sens, de nouvelles manières de faire, de nouvelles stratégies, de nouveaux coups, etc. Tout jeu présente à la fois l'incertitude des possibles et l'incertitude de la part virtuelle que le joueur peut s'approprier : plus l'incertitude de la part virtuelle est grande, plus l'espace d'appropriation en puissance est large.

21 En fait, pour qu'une structure-jeu soit jouée, elle doit nécessairement laisser un espace d'appropriation au joueur, aussi restreint soit-il. Le joueur ne joue pas qu'avec des possibles, mais avec ce flottement de sens des différentes variables de l'ensemble du jeu. Le sens du jeu n'est pas préalablement donné au joueur, mais créé par celui qui s'approprie le jeu. Cette création permise grâce à l'actualisation de la part virtuelle donne un sens nouveau par rapport à ce qui était délimité par les possibles. "Arrive toujours un instant où le calcul cède le pas à l'improvisation, où la réflexion sage et méthodique fait place à l'alea jacta est" (Henriot, 1989, p. 239). Il y a à la fois improvisation du sens à donner aux signes et improvisation des moyens. Dans les jeux codifiés (ludus), la marge laissée à l'improvisation, c'est-à-dire au virtuel, est moins grande que dans les jeux peu codifiés (paidia) où l'improvisation, la création, devient le moteur principal du jeu.

Néanmoins, dans tous les types de jeux existe une certaine incertitude liée au virtuel de la structure. Même dans le poker ou les échecs existe cette part de flottement, d'improvisation où le joueur improvise, marque son style de jeu. À partir de la somme des connaissances « cachées ", comme le dit Henriot (1989, p. 239), le joueur se ménage un espace d'appropriation pour donner le sens unique de son expérience ludique. 
Aucun jeu n'est entièrement prédéfini. Un certain nombre de possibles peuvent être définis, mais une part virtuelle n'est pas définissable avant qu'il y ait eu actualisation (toujours du point de vue du joueur). Cette part est plus ou moins grande selon la manière de jouer du joueur (davantage paidia ou ludus, avec plus d'improvisation ou plus de calculs). Le virtuel est le « point de fuite » que le joueur peut tracer à travers les règles du jeu pour produire un sens nouveau et ne pas se limiter à une répétition des possibles du jeu.

23 Ainsi, le virtuel est toujours en excès par rapport aux possibles, car il ajoute une nouvelle occurrence à ce qui était "possible». L'actualisation de la part virtuelle augmente l'éventail des possibles en venant ajouter un nouveau possible jusqu'à présent inconnu (de la part du joueur). Cette plus-value est illimitée puisque l'on ne saurait la restreindre sans en faire des possibles. Par définition, elle ne peut pas être énumérée, ni décrite ou imaginée: si elle le pouvait, elle s'incorporerait déjà aux possibles (le virtuel est d'ailleurs toujours au singulier : il n'y a pas des virtuels, mais un virtuel indéfini). Le virtuel $\mathrm{du}$ jeu est la part indéfinissable que le joueur peut s'approprier en tant que paidia. C'est la marge donnée aux joueurs pour définir leur style de jeu et même faire évoluer certains jeux grâce à une interprétation nouvelle des règles. C'est, par exemple, Dick Fosbury qui, lors des Jeux Olympiques de 1968, saute pour la première fois de manière dorsale au lieu de sauter en ciseau. L'action, désormais appelée le Fosbury-flop, était virtuellement présente, mais à partir du moment où un sauteur l'actualise, il en fait une possibilité pour tous les autres sauteurs. On comprend alors que le jeu se reconfigure sans cesse grâce à cette liberté interprétative : le jeu est nécessairement créatif, sinon il n'y a plus de jeu. Tout n'est pas défini, surtout pas le sens du jeu et du joueur, et l'actualisation de la part virtuelle est la fonction de jeu. Ainsi, le Fosbury-flop est intégré dans les nouvelles règles du jeu et, aujourd'hui, presque tous les sauteurs utilisent cette technique. ${ }^{5}$

\section{La distance est ce qui permet la création}

Ainsi faut-il comprendre Henriot lorsqu'il affirme que l'artiste et le technicien laissent une place au jeu au moment de produire œuvres et biens. «Par leur audace et une certaine perte de contrôle » sur leur production, ils introduisent une part de jeu dans leur travail et cette part est la part proprement créative. C'est le flottement de l'interprétation qui introduit de la différence dans le monde grâce à l'acte créatif. Ces "producteurs » ont besoin du jeu pour créer et le jeu constitue probablement leur principale motivation. Le jeu est productif (Henriot, 1989, p. 173) puisqu'il introduit de nouvelles occurrences qui sont par la suite inclues dans l'ordre du possible.

La distance de Henriot peut ainsi être définie comme cette part de créativité indéfinie et illimitée du virtuel. Selon Henriot, réduire la part d'incertitude est réduire d'autant la part de jeu (Henriot, 1989, p. 244), mais nous ajoutons que réduire la part d'incertitude issue du virtuel est réduire d'autant la distance. Plus la contingence issue $\mathrm{du}$ virtuel est grande, plus la distance est large, car plus le joueur peut particulariser le sens de son jeu en créant de nouveaux possibles par des rapports différents aux variables. Le joueur peut s'approprier la contingence du jeu et faire différer le sens du jeu en ajoutant de nouvelles formes. Dans un jeu comme le morpion ou Angry Birds, ${ }^{6}$ la distance est étroite et la part de virtuel est limitée par la structure du jeu, mais dans le jeu de Go ou dans Guild Wars $\mathrm{II}^{7}$, cette distance est large et permet une particularisation 
de l'expérience ludique grâce à l'appropriation de la part virtuelle du jeu. La distance de Henriot est cet espace d'appropriation, cette part de virtualité que le joueur actualise ou non - tout dépend des modes de rapport au jeu dans lesquels le joueur entrera.

En effet, la question est alors de savoir ce que fait le joueur de cette distance. Actualiser les possibles du jeu et répéter ce qui a déjà été perçu ou conçu, ou actualiser une part virtuelle du jeu et s'approprier le jeu pour créer un sens unique à son expérience (grâce à l'effort, l'imagination, la créativité, etc.) dépend de l'interprétation que fera le joueur du jeu. Il peut y avoir une distance, un vide, un espace d'appropriation en puissance, encore faut-il que le joueur l'actualise. Il faut établir une importante distinction entre ce qui est en puissance et l'actualisation effective du jeu. Tous les joueurs ne font pas le même usage de leur liberté interprétative et certains joueurs peuvent même s'approprier le jeu de telle manière qu'au lieu d'élargir leur espace de jeu, ils sont davantage contraints. Bien que toutes les appropriations soient l'expression de la liberté interprétative, elles ne sont pas toutes bénéfiques pour le joueur. Elles peuvent finalement réduire l'espace de jeu, la distance entre le joueur et le jeu. Il faut se soucier du jeu, mais plus précisément, il faut se soucier de son rapport au jeu.

En effet, l'actualisation d'une part virtuelle pousse le joueur à se questionner sur la valeur de ses actes, car ses interprétations représentent les premières occurrences d'une manière de jouer (pensons ici au Fosbury-flop). Si un jugement clair est posé sur les actions qui transgressent les règles dans l'ordre des possibles, il en va autrement dans l'ordre du virtuel où il peut y avoir invention d'occurrences nouvelles et réinvention des règles. Le jeu est ce que le joueur crée comme sens et il appartient finalement au joueur de déterminer le genre de rapport dans lequel il veut entrer en jouant. Si le joueur définit l'espace de jeu par l'actualisation des possibles ou de la part virtuelle du jeu, il a donc une responsabilité dans le sens produit et dans la forme du jeu qui est partagée.

Le joueur doit donc porter un jugement sur le jeu qu'il actualise, sur la forme de la distance qui s'actualise, mais ce jugement ne concerne pas seulement le sens produit par ses actes, mais le sens de lui-même. Le jeu est « idée d'homme ", mais "l'idée de jeu est en même temps une idée de l'homme : idée qu'il se fait lui-même de ce qu'il est, de ses pouvoirs" (Henriot, 1989, p. 158). Si le joueur décide de tricher, il devient, littéralement, un tricheur; si le joueur décide de perfectionner le jeu grâce à une maitrise exceptionnelle, il devient un expert. Gadamer ajoute ainsi que « le sens de son jeu est aussi une pure représentation de soi » (1960, p. 123). Jouer n'est en rien insignifiant: en tant que terme relatif culturellement, le sens du jeu est une représentation du sens de soi-même et, considéré comme une fonction, le jeu permet à l'individu et au monde de se renouveler grâce à l'actualisation d'une part du virtuel. Le joueur est responsable du sens du jeu produit dans le monde pour lui-même et pour les autres. De nombreux liens unissent le jeu à l'éthique : jouer est toujours une action et un acte éthique, l'éthique étant entendue comme l'art de diriger la conduite.

29 Le joueur doit faire l'effort d'entrer dans un rapport de composition avec le jeu et les autres joueurs pour pouvoir élargir son espace de jeu, c'est-à-dire la distance entre lui et le jeu. Le joueur qui respecte les règles (telles qu'interprétées par les joueurs) joue avec les autres et entre en composition avec la communauté des joueurs. Or, cet espace de jeu sera d'autant plus grand si le joueur, dans son rapport de composition, s'approprie en plus la part virtuelle du jeu. Le joueur qui actualise une part virtuelle 
fait preuve d'imagination lors de l'expérience ludique et agrandit son espace de jeu en créant de nouveaux possibles pour lui-même, mais aussi pour tous les autres joueurs.

Nous posons alors l'hypothèse que l'effort de s'approprier la part virtuelle du jeu ou, en d'autres mots, de cultiver cette nécessaire distance entre le joueur et le jeu, constitue un idéal éthique. Tout joueur doit faire un travail constant pour garder la distance dont parle Henriot : le joueur doit se ménager un espace de jeu et garder une distance par rapport à ce qu'il fait. «Si l'on pénètre dans le jeu au point de n'avoir plus de possibilité de recul et de désengagement, si l'on joue au point d'en venir à oublier qu'on joue, alors on se trouve aliéné : on ne joue plus » (Henriot, 1969, p. 88). Ainsi, le joueur doit faire l'effort de ne pas se laisser prendre au jeu et ne plus avoir d'espace de mouvement.

Entre le moment où, par détachement, on ne joue pas et celui où, par application, on ne joue plus, où situer l'instant infinitésimal où l'on joue ? Il y a là un emplacement qui ne tient pas de place, où nul ne peut séjourner, s'installer, prendre son temps (Henriot, 1989, p. 149).

Pour jouer, il faut constamment faire un effort et la technique consiste justement à développer cette capacité à garder une distance constante. Ce travail de distanciation fait partie de l'éthique du joueur et doit guider sa conduite :

c'est le joueur qui, par la pensée, se déplace, change de position par rapport au monde qui l'entoure et à lui-même. Adopte un point de vue différent du point de vue habituel, met les choses et se met lui aussi en perspective (Henriot, 1989, p. 264).

Le moyen d'élargir son espace de jeu pour se mettre à distance est de s'approprier la part virtuelle du jeu. Tous les joueurs n'usent pas de leur liberté interprétative de la même manière et certains joueurs qui adoptent une manière de jouer près du ludus sont disciplinés et suivent les possibles. Cependant, d'autres adoptent la paidia comme manière de jouer et improvisent de nouvelles interprétations qui augmentent le spectre des possibles et, donc, les possibilités d'être affecté. Ces joueurs ne se conforment pas à l'interprétation commune du jeu parce qu'ils font un effort constant de se mettre à distance de façon renouvelée en introduisant une part virtuelle du jeu. En ce sens, la pratique ludique peut devenir une occasion unique de se réaffirmer en tant qu'individu. De faire l'exercice de la distance, de cultiver la distance dont parle Henriot peut devenir une technique de soi qui permet de réintroduire une liberté interprétative dans sa vie de tous les jours.

\section{BIBLIOGRAPHIE}

BONENFANT M. (2010), « Le jeu comme producteur culturel : distinction entre la notion et la fonction de jeu », in Gadbois J. (dir.), Ethnologies, CELAT - Université Laval (Québec), pp.51-69. BONENFANT, M. (2011), « Les mondes numériques ne sont pas “virtuels" : l'exemple des jeux vidéo en ligne », Revue des Sciences Sociales, 45, pp. 60-67.

CAILLOIS R. (1967), Les jeux et les hommes : le masque et le vertige [1958], Paris, Gallimard

FINK E. (1965), Le jeu comme symbole du monde [1960], Paris, Éditions de Minuit, coll. Arguments. 
GADAMER H-G. (1996), Vérité et méthode : les grandes lignes d'une herméneutique philosophique [1960], Paris, Seuil, Coll. L'ordre philosophique.

HENRIOT J. (1969), Le jeu, Paris, PUF, coll. Sup Initiation philosophique.

HENRIOT J. (1989), Sous couleur de jouer : la métaphore ludique, Paris, José Corti.

HOCK-KOON S. (2011), « La ruse, la triche et la règle dans le jeu vidéo », in Perraton, C. et Bonenfant M. (dir.), La ruse : Entre la règle et la triche, Presses de l'Université du Québec, pp.97-112.

SALEN K. \& ZIMMERMAN E. (2004), Rules of Play: Game Design Fundamentals, Cambridge, The MIT Press.

SUITS B. (1990), Grasshopper: Games, Life, and Utopia [1978], Boston, David R. Godine.

\section{NOTES}

1. Cet article sera partiellement publié dans le livre S'approprier World of Warcraft : penser le concept d'appropriation dans les jeux vidéo en ligne, aux Presses de l'Université de Montréal (à paraître).

2. Dans une grille à neuf cases, deux joueurs doivent, à tour de rôle, mettre un " $\mathrm{x}$ " ou un « $\mathrm{O}$ " afin de compléter une ligne verticale, horizontale ou diagonale de trois éléments identiques. Le premier à compléter une ligne gagne la partie.

3. Nous reprenons ces deux dénominations à Caillois (1967).

4. Bernard Suits (1978) parle de la lusory attitude.

5. Sébastien Hock-Koon (2011) développe plus en détail cet exemple dans l'article « La ruse, la triche et la règle de jeu vidéo ».

6. Angry Birds (2009) est un jeu vidéo basé sur la résolution d'un puzzle relativement simple et similaire à chaque tableau où le joueur doit détruire une structure par l'envoi de projectiles.

7. Guild Wars II (2012) est un jeu vidéo en ligne massivement multijoueur où le joueur contrôle un avatar qui accomplit différentes actions et évolue dans un monde persistant.

\section{RÉSUMÉS}

Jacques Henriot affirme que le jeu ne fait référence qu'à une seule et même idée. Il croit en un noyau sémantique, un élément commun, une « unité du jeu» parmi la diversité des jeux. Ce «dénominateur commun », selon l'expression de Caillois, est ce qui fait qu'on joue dans tous les types de jeux. Ce noyau sémantique est, selon notre lecture de Henriot, la « distance » qui définit la fonction de jeu, car l'idée de jeu doit d'abord se comprendre d'une façon mécanique. Le jeu est avant tout ce vide qui permet de dire qu'il y a du jeu: dans les pièces d'un engrenage, dans les jointures, dans une penture, etc. Jouer est alors considéré comme une opération dialectique qui met à distance le joueur. La forme du jeu est cette distance, cet intervalle qui permet au jeu d'exister pour un joueur. La « distance » est donc un concept-clé pour comprendre la conception du jeu chez Henriot, mais au lieu d'aborder la «distance» d'un point de vue psychanalytique, nous proposons de définir ce concept à partir du concept de "virtualité » pour bien saisir la richesse de la réflexion de Henriot. 
Jacques Henriot states that play refers to one same and single idea. He believes in a semantic core, a common element, one play unit amongst the diversity of games. This «common denominator ", as called by Caillois, explains why one plays in any kind of games. Referring to Henriot, our understanding is that this semantic core is the "distance " which defines the function of play, because the concept of play must first be understood in a mechanical way. Play is foremost that space (empty gap) which allows to state that there is a «kind » of play: in parts of a gear, in joints, in hinges, etc. Play is then considered as a dialectical operation that puts the player at a distance. The nature of play is this distance, this gap that creates the game for a player. Distance is thus a key-concept to understand Henriot's conception of play, but instead of considering this « distance » in the psychoanalytical context, we aim to define it with the concept of « virtuality », to better encompass Henriot's fertile thought.

INDEX

Mots-clés : jeu mécanique, distance, virtualité, fonction, forme du jeu, création, éthique

Keywords : mechanical play, distance, virtuality, function, play form, creation, ethics

\section{AUTEUR}

\section{MAUDE BONENFANT}

Université du Québec à Montréal 